\title{
Spontaneous Photon Production in Time-Dependent Epsilon-Near-Zero Materials
}

\author{
A. Prain, S. Vezzoli, N. Westerberg, T. Roger, and D. Faccio \\ Institute of Photonics and Quantum Sciences, School of Engineering and Physical Sciences, \\ Heriot-Watt University, EH14 7AS Edinburgh, United Kingdom
}

(Received 23 October 2016; published 29 March 2017)

\begin{abstract}
Quantum field theory predicts that a spatially homogeneous but temporally varying medium will excite photon pairs out of the vacuum state. However, this important theoretical prediction lacks experimental verification due to the difficulty in attaining the required nonadiabatic and large amplitude changes in the medium. Recent work has shown that in epsilon-near-zero (ENZ) materials it is possible to optically induce changes of the refractive index of the order of unity, in femtosecond time scales. By studying the quantum field theory of a spatially homogeneous, time-varying ENZ medium, we theoretically predict photon-pair production that is up to several orders of magnitude larger than in non-ENZ time-varying materials. We also find that while in standard materials the emission spectrum depends on the time scale of the perturbation, in ENZ materials the emission is always peaked at the ENZ wavelength. These studies pave the way to technologically feasible observation of photon-pair emission from a time-varying background with implications for quantum field theories beyond condensed matter systems and with potential applications as a new source of entangled light.
\end{abstract}

DOI: 10.1103/PhysRevLett.118.133904

Introduction.-Epsilon-near-zero (ENZ) materials are characterised by relative dielectric permittivity, whose real part, $\varepsilon_{r}$, attains near-zero values around a given frequency $\omega_{\mathrm{ENZ}}[1,2]$. A natural example of ENZ materials are commercially available transparent conducting oxides, e.g., indium-tin-oxide (ITO) or Al-doped $\mathrm{ZnO}$ (AZO), where $\varepsilon_{r}$ crosses 0 near the plasma frequency, whereas the imaginary part $\varepsilon_{i}$ is small. These materials are attracting attention for their rather remarkable properties, ranging from a geometric invariance of resonant structures to novel light propagation regimes and light emission geometries [2-10]. Recent work pioneered by Engheta et al. has started to focus on the quantum properties of these materials, including quantum emission in ENZ cavities and limitation or even complete suppression of vacuum modes $[11,12]$.

Alongside the fascinating linear optical properties of these materials, the nonlinear optical response exhibits an enhancement for frequencies close to $\omega_{\mathrm{ENZ}}$ [13-19]. In these first studies, the main enhancement mechanism of the nonlinearity is related to an enhancement of the $z$ component (directed along the optical propagation axis) of the electrical field of the intense optical pump beam.

An alternative nonlinear enhancement mechanism has also been reported that is based on the simple realization that in the ENZ region even a small change of permittivity $\Delta \varepsilon$, induced by a third-order Kerr nonlinearity, can result in a

Published by the American Physical Society under the terms of the Creative Commons Attribution 4.0 International license. Further distribution of this work must maintain attribution to the author(s) and the published article's title, journal citation, and DOI. large relative change of the refractive index $\Delta n / n_{0}$, as a result of the small linear refractive index $n_{0}$ [20,21]. Indeed, for materials such as ITO and AZO that also exhibit a small imaginary part of the permittivity, $\varepsilon_{i}$, the real part of the refractive index $n_{0}$ is also close (although never equal) to 0 around $\omega_{\mathrm{ENZ}}$; thus, $\Delta n / n_{0}$ can easily attain values of the order of unity, compared to the modest $10^{-4}-10^{-3}$ values of non-ENZ materials [20,21]. Remarkably these huge changes are also ultrafast, with rise times below the duration ( $\sim 100 \mathrm{fs})$ of the laser pulse used to induce the nonlinear response.

The main underlying concept of this work is that the ultrafast and order-of-unity refractive index change in ENZ materials provides access to the quantum physics of spatially homogenous media with a time-varying parameter.

Quantum field evolution on a time-dependent background is a hallmark problem in quantum field theory (QFT) in curved spacetimes. One of oldest and most robust predictions of QFT is that a homogeneously expanding universe will excite entangled photon pairs from the quantum vacuum state [22-26]. Although this kind of particle production is believed to be dominant in the very early Universe and is argued to be responsible for the initial seeds of inhomogeneity, which gave rise to the visible structures of the Universe we live in [27-29], no experimental evidence of this has been observed so far. A similar effect is predicted to occur also in condensed matter systems [30] including Bose Einstein condensates [31-33], rings of trapped ions [34], superfluid gases [35], and nonlinear optics [36]. There is a specific and independent body of literature concerned with the classical and quantum consequences of a homogeneous time variation of the real part of the refractive index alone 
under the name "time refraction" [37,38]. Perhaps the first work in this general area is the work of Yablanovitch from 1989 [39].

In this work, we consider the specific case of the optical nonlinear Kerr effect in an ENZ material, such that a passing pulse of intense light modifies the refractive index of the background material on top of which vacuum fluctuations are propagating. Using methods of QFT that have previously been applied in the context of expanding cosmologies, we explicitly calculate the photon-pair production in ITO close to the ENZ frequency window. We show an enhancement of 7 orders of magnitude compared to non-ENZ Kerr media and demonstrate that the emission spectrum always shows a peak emission close to the ENZ wavelength (1377 $\mathrm{nm}$ in our studies). Taking losses into account by including the imaginary part of $n$, using the data of Ref. [40] we predict roughly $10^{-4}$ photon pairs per pump pulse in $1 \times 1 \times 1 \mu \mathrm{m}^{3}$ of ITO, which is in the detectable region of current single photon detectors.

QFT model.-We first illustrate how particle production emerges in a standard nonlinear medium as a consequence of a time variation of the dielectric environment induced by a pump beam. In a spatially homogeneous material (i.e., pumped with a spatially uniform laser beam) that is changing in time, the $k$ vector is conserved and the optical frequency $\omega$ is modified. Spatial homogeneity allows one to separate the partial differential wave equation governing the full quantum field into a collection of ordinary differential equations labeled by $k$,

$$
\frac{\partial^{2}}{\partial t^{2}} E-c^{2} \nabla^{2} E \Rightarrow \frac{d^{2} E_{k}}{d t^{2}}+\omega_{k}^{2}(t) E_{k}=0 .
$$

Equation (1) describes the evolution of a single fixed momentum mode $E_{k}(t)$ of a full quantum field $E(t, x) \propto$ $\int e^{-i k x} E_{k}(t)$ and here the time dependence of $\omega_{k}(t)=$ $c k / n(t)$ is due to a time-dependent refractive index $n(t)=n_{0}+\Delta n(t)$, with $\Delta n(t)=n_{2} I(t)$, where $n_{2}$ is the nonlinear Kerr index and $I(t)$ is the intensity profile of the laser pump pulse [40]. We also assume that the medium is pumped orthogonally so that the time variation is spatially uniform in the $x-y$ plane: photons are then produced in this plane, as schematically summarized in Fig. 1.

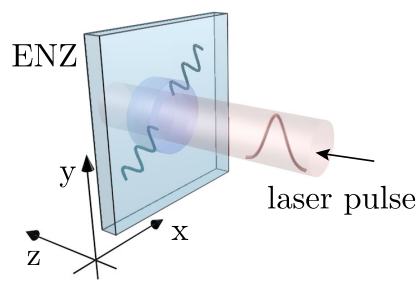

FIG. 1. Schematic layout of the interaction geometry: an intense laser pulse directed along the $z$ axis uniformly illuminates a region of the ENZ material. Photons are produced in the $x-y$ plane in which the time variation is spatially uniform.
The functions $\omega_{k}(t)$ are constant, $\omega_{k}=c k / n_{0}$, well before $(t \rightarrow-\infty)$ and after $(t \rightarrow \infty)$ the nonlinear interaction and change quickly in a small time window related to the pump pulse duration. The notion of particle or photon is only well defined in regions for which $\omega_{k}(t)$ is approximately constant $\dot{\omega}_{k} / \omega_{k} \ll 1$, i.e., for modes in which the time variation is adiabatic, whereas in the intermediate regime, when $\omega_{k}(t)$ is varying rapidly, the notion of a photon is approximate or absent altogether [41]. Very generically speaking, an initial configuration of welldefined photon number, when propagated through a (time) region in which the photon number is not defined can, and in general will, emerge into the second region where photon number is again well defined but with a different number of photons (see for example Ref. [23] for an overview).

For each momentum $k$ it is possible to calculate the total number of produced photons of momentum $k$ by solving the equation of motion Eq. (1) with a purely positive frequency initial condition. This input condition evolves into a linear combination of positive and negative frequencies,

$$
\frac{e^{-i \omega_{k} t}}{\sqrt{2 \omega_{k}}} \stackrel{t \rightarrow-\text { infty }}{\longrightarrow} E_{k}(t) \stackrel{t \rightarrow+\infty}{\longrightarrow} \alpha_{k} \frac{e^{-i \omega_{k} t}}{\sqrt{2 \omega_{k}}}+\beta_{k} \frac{e^{+i \omega_{k} t}}{\sqrt{2 \omega_{k}}} .
$$

The Bogoliubov coefficient $\beta_{k}$ is the coefficient of the negative frequency component of the solution at late times. The number of produced photons for a given $k$ is then directly proportional to the square of the Bogoliubov coefficient $\left|\beta_{k}\right|^{2}$ [23].

For illustration, consider Eq. (1) with a time-dependent frequency given by $\omega_{k}(t)=c k / n(t)$ with refractive index $n(t)=1+\delta \operatorname{sech}^{2}(t / \tau)$ where $\delta=\Delta n / n_{0}$. In this case the Bogoliubov spectrum admits an analytical solution (see Supplemental Material) and takes the shape shown in Fig. 2: the emitted photon spectrum has a peak that shifts to the infrared for increasing rise time $\tau$ of the time variation, where single or few photon detection is extremely challenging or currently not possible. In the

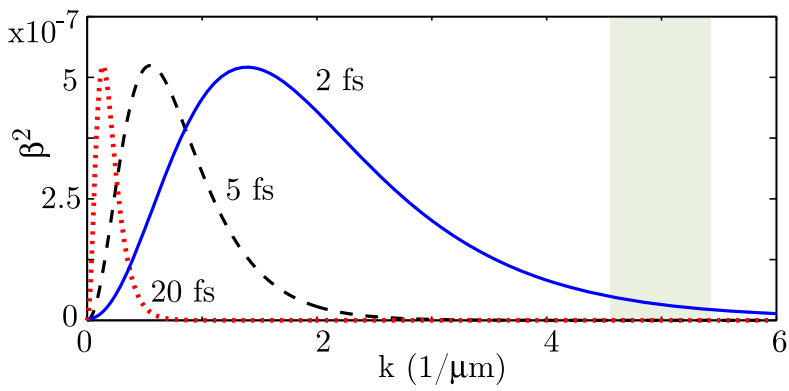

FIG. 2. The spectrum of produced photons $\left(\alpha \beta^{2}\right)$ in the dispersionless case $\left(n_{0}=1\right)$; the material response is assumed constant in $k$ and such that $\Delta n / n_{0}=10^{-3}$. Our equations reproduce the standard result showing how the peak of the spectrum shifts to the infrared for increasing time-variation rise time $\tau$, indicated in the figure. 
experimentally feasible detection region (for example, in the green shaded region, taken as the same ENZ region examined in more detail below) the amplitude $\left|\beta_{k}\right|^{2}$ is exponentially suppressed. Finally, the maximum number of photons is typically extremely small also because the maximum amplitude of $\left|\beta_{k}\right|^{2}$ scales with the (squared) variation of the medium, $\delta^{2}$ (see Supplemental Material [42]), which is very small $\left(\delta^{2} \simeq 10^{-6}\right)$ in typical nonlinear media. All combined, these known results lie at the heart of the problem of actually detecting photon emission from a standard time-dependent medium.

Photon-pair creation in the ENZ region.-In an ENZ material, close to the ENZ frequency it is possible to use a simple resonance-free Drude-Lorentz model for the linear permittivity $[15,21]$,

$$
\varepsilon^{\prime}+i \varepsilon^{\prime \prime}=\varepsilon_{\infty}-\frac{\omega_{p}^{2}}{\omega^{2}+i \omega \Gamma},
$$

where $\varepsilon^{\prime}$ and $\varepsilon^{\prime \prime}$ are the real and imaginary parts of the permittivity, $\Gamma$ is related to the electron damping in the material, and $\omega_{p}$ is the plasma frequency. Essential in this work is the existence of a zero crossing for the real part of the permittivity, which occurs at approximately $\omega_{\mathrm{ENZ}} \simeq \sqrt{\omega_{p}^{2}-\Gamma^{2}}$. In Ref. [15], experimental values for an ITO are reported as $\varepsilon_{\infty} \simeq 4.082, \omega_{p}^{2} \simeq$ $7.643 \times 10^{30} \mathrm{~s}^{-1}$, and $\Gamma \simeq 1.239 \times 10^{14} \mathrm{~s}^{-1}$ leading to $\omega_{\mathrm{ENZ}}$ at around $1.36 \times 10^{15} \mathrm{~s}^{-1}$ or, in terms of wavelength, at $1377 \mathrm{~nm}$. Near the ENZ region, the real part of the refractive index defined through $n_{0}=n_{0 r}+i n_{0 i}=\sqrt{\varepsilon^{\prime}+i \varepsilon^{\prime \prime}}$ is also close to 0 (but never exactly 0 due to the nonzero imaginary permittivity), as shown in Fig. 3.

In a dielectric dispersive medium, it is possible to reduce the full wave equation to the study of equations of the form (1) (see Supplemental Material [42]),

$$
\frac{\partial^{2}}{\partial t^{2}} E_{k}+\omega_{k}^{2} E_{k}=0, \quad \omega_{k}^{2}=\frac{c^{2} K^{2}}{\epsilon^{\prime}+i \epsilon^{\prime \prime}},
$$

where $K$ is the material wave number, which is conserved during the time evolution. Expressed in terms of the vacuum wave number, the material wave number is $K(k)=k\left[n_{0 r}(c k)+i n_{0 i}(c k)\right]$, where $n_{0 r}$ and $n_{0 i}$ are the constant, real, and imaginary parts of the background refractive index. Hence, the relevant dynamical equation is of the form Eq. (1) with

$$
\omega_{k}(t)=\frac{c K(k)}{n_{r}(c k)+i n_{i}(c k)} .
$$

The time dependence in the frequency of Eq. (5) is given by the nonlinear change of the refractive indices that appear in the denominator. In particular, the real part of the refractive index $n_{r}$ is modified due to the pump laser pulse
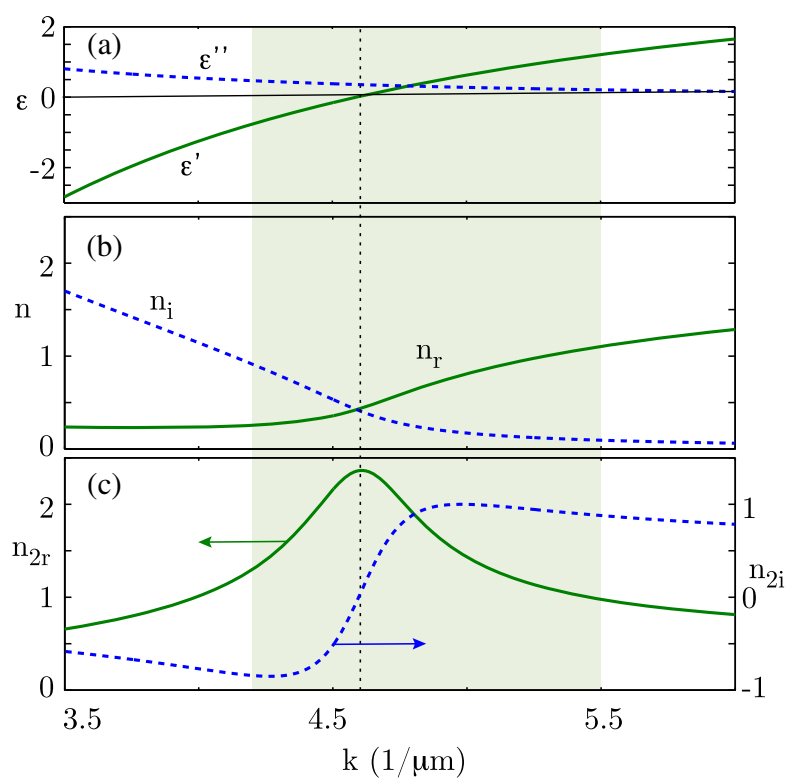

FIG. 3. (a) Dielectric permittivity and (b) refractive index for ITO. (c) The nonlinear refractive indices are represented by the proportionality factors $n_{2 r} \propto\left(n_{r}+n_{i}\right) /\left(n_{r}^{2}+n_{i}^{2}\right)$ and $n_{2 i} \propto\left(n_{r}-n_{i}\right) /\left(n_{r}^{2}+n_{i}^{2}\right)$. The shaded area is the ENZ region, here taken as as $\left|\varepsilon^{\prime}\right|<1$. The vertical dotted line indicates the ENZ frequency.

via the nonlinear Kerr effect so that $n_{r}(t)=n_{0 r}+n_{2 r} I(t)$ where $I(t)$ is the local intensity of the pump beam and $n_{2 r}$ is the real part of the nonlinear Kerr index. We recall that in ENZ materials the nonlinear response is strongly affected by the linear dispersion [20]. As a consequence, the nonlinear change of the real part, $\Delta n_{r}=n_{2 r} I(t)$, can be of the order of unity. Assuming that $\chi^{(3)}$ has no dispersion, and has equal real and imaginary parts, $n_{2 r} \propto D=\left(n_{0 r}+n_{0 i}\right) /\left(n_{0 r}^{2}+n_{0 i}^{2}\right)$, which is peaked around the ENZ frequency [20] (see Fig. 3).

The frequency given by Eq. (5) is real valued for early and late times where it reduces to $\omega_{k}=c k$. However, during the time variation, $I(t) \neq 0$ and therefore $\omega_{k}(t)$ becomes complex. The refractive indices $n_{r}$ and $n_{i}$ both enter in the real and imaginary parts of $\omega_{k}(t)$; including $n_{i}$ leads to a modification of the particle production spectrum [governed by the real part of $\omega_{k}(t)$ ] and to a time modulated absorption [governed by the imaginary part of $\omega_{k}(t)$ ]. We note that $n_{2 i} \propto\left(n_{0 r}-n_{0 i}\right) /\left(n_{0 r}^{2}+n_{0 i}^{2}\right)$ [20], which therefore is 0 exactly at the ENZ frequency $\left(n_{0 r}=n_{0 i}\right.$; see Fig. 3(c)). Its effects are therefore felt inside the ENZ region but not at the exact ENZ frequency.

In the following, we model the laser pump pulse and hence the temporal variation profile with a $\operatorname{sech}^{2}(t / \tau)$ function and we numerically calculate the spectrum for various rise times $\tau$ with a positive frequency input condition as indicated in Eq. (2).

Results.-As a first calculation, let us assume that $n_{i}=0$. The numerical results are shown in Fig. 4 . 


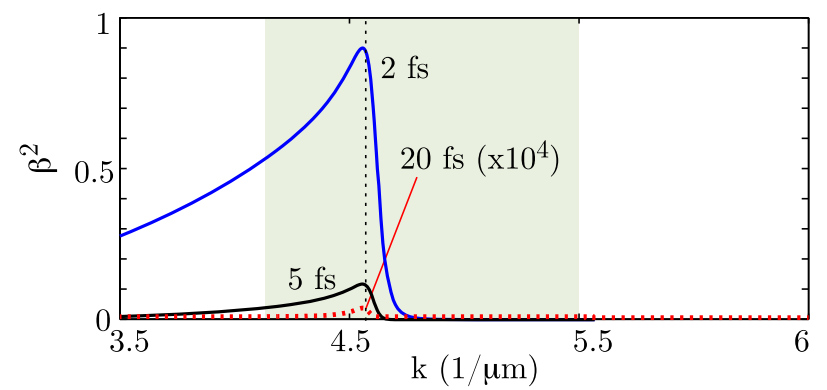

FIG. 4. The spectrum of produced photons in an ENZ material from the full calculation assuming that the imaginary part of the refractive index is 0 . The shaded region coincides with the ENZ region of Fig. 3. Different rise times are indicated in the figure. The vertical dotted line indicates the ENZ frequency.

Comparing to Fig. 2, we note a first and obvious difference: although in both figures we use the same values for the time-variation rise times ( $\tau=2,5,20 \mathrm{fs})$, in the case of an ENZ material the typical and expected redshift of the photon emission peak for increasing $\tau$ is completely absent. Rather, we find a new emission peak that is now locked onto the ENZ frequency. This is somehow reminiscent of a similar frequency locking observed for resonant antennas placed in proximity to an ENZ substrate $[44,45]$ and is of crucial importance for future experiments: ENZ allows one to separate the temporal rise time of the pump laser pulse from the peak spectral emission wavelength. In this way, longer and technologically accessible laser pulses can be used in combination with the possibility to match the emission to the photon detection sensitivity (typically centered around 700 or $1400 \mathrm{~nm}$ wavelengths). We also underline the significant enhancement in the emissivity that is now close to 7 orders of magnitude better if we compare the absolute maxima in both cases but is actually many orders of magnitude larger if we restrict the comparison to the ENZ window, and for pump laser pulses with technologically feasible durations of 5 fs or larger.

In Fig. 5 we show the results using the full complex refractive index, with real and imaginary parts shown in Fig. 3(b). The maximum values of $\left|\beta_{k}\right|^{2}$ are sightly

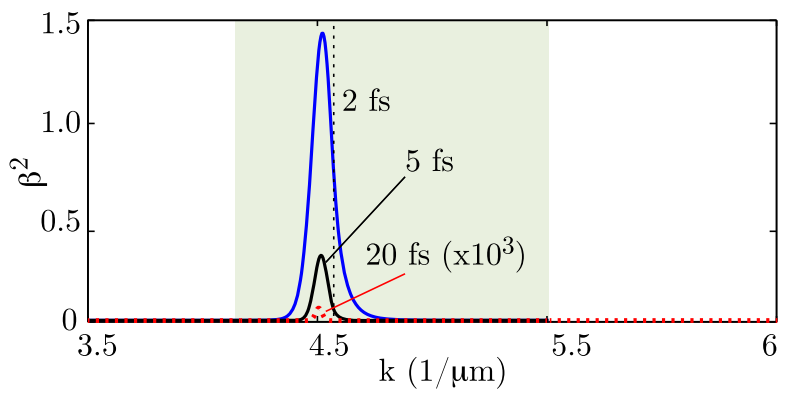

FIG. 5. The spectrum of produced photons in an ENZ material from the calculation with the full complex refractive index shown in Fig. 3(b). Different rise times are indicated in the figure. enhanced by the additional contribution of $n_{i}$. The peak is now also relatively localized around ENZ due to the fact that the real part of $\omega_{k}$ that is responsible for photon production depends on both $n_{r}+n_{2 r}(t)$ and $n_{i}+n_{2 i}(t)$ (see Supplemental Material [42]). Therefore, for frequencies above ENZ, the time variation of $\omega_{k}$ occurs on a high $n_{r}$ background. For low frequencies, the time variation occurs on a high $n_{i}$ background (see Fig. 3). Only very close to ENZ, the time variation is enhanced by a simultaneously low $n_{r}$ and $n_{i}$. A smaller effect is also visible: the peak emission is slightly shifted to lower frequencies with respect to the ENZ frequency (vertical dotted line). This is a result of the $n_{i}$ time-varying contribution being equal to 0 exactly at the ENZ frequency and asymmetric around ENZ.

In terms of the number of photons actually emitted, we must account also for propagation absorption of these photons. This leads to an additional exponential damping term that restricts the allowed physical size of the medium. For example, if we consider a $1 \times 1 \times 1 \mu \mathrm{m}^{3}$ volume, then the effects of losses amount to a $\sim 10 \times$ reduction factor with respect to the lossless case. We therefore predict an actual photon emission spectrum of $N_{k} \sim 10^{-6}\left|\beta_{k}\right|^{2}$ per pump laser pulse (see Supplemental Material [42]): a 5 fs (10 fs when measured at full width) laser pulse is expected to produce $\sim 10^{-7}$ photon pairs at the ENZ wavelength per laser pulse, which implies a $\sim 10 \mathrm{~Hz}$ emission rate using a $100 \mathrm{MHz}$ laser source. These photons could then be extracted from the thin film, either by a grating coupler or by direct coupling to a waveguide structure.

Conclusions. - ENZ materials exhibit a series of novel nonlinear features that lead to a dramatically enhanced interaction with the quantum vacuum. We demonstrate a 7 orders of magnitude enhancement of the quantum amplification of vacuum fluctuations for (vacuum) frequencies close to the ENZ region, i.e., in the near IR. We describe a scenario where the detection of pairs of entangled photons should be experimentally feasible.

The vacuum-seeded photon emission due to a timevarying ENZ medium presented here is very generic. It is independent of the specific choice of ENZ material and functional shape of the time variation; these parameters may be tuned to increase the expected photon emission rate although we preferred to choose physically relevant parameters, i.e., as measured for a real material. The emission at the ENZ wavelength should be easily distinguishable from other effects such as self-phase modulation on the pump pulse (by pumping at a distant wavelength) but fluorescence emission needs to be assessed to ensure that it is negligible in the ENZ region. We also note that, in keeping with the standard quantum optics description $[46,47]$, a nonzero $\beta_{k}$ Bogoliubov coefficient implies optical amplification; i.e., a (classical laser pulse) seeded amplification process could be used to assess the effects of the timevarying ENZ material. 
A.P. acknowledges the support from the People Programme (Marie Curie Actions) of the European Union's FP7 Programme, Grant No. 659301 (SOUNDCONE) and S. Seahra for help with the numerical code. D. F. acknowledges financial support from the European Research Council under the European Union Seventh Framework Programme Grant No. (FP/20072013)/ERC GA 306559 and Engineering and Physical Sciences Research Council (UK, Grants No. EP/ M009122/1, No. EP/L015110/1).

[1] N. Engheta, Science 340, 286 (2013).

[2] I. Liberal and N. Engheta, Opt. Photonics News 27, 26 (2016).

[3] I. Liberal, A. M. Mahmoud, and N. Engheta, Nat. Commun. 7, 10989 (2016).

[4] J. Soric, N. Engheta, S. Maci, and A. Alu, IEEE Trans. Antennas Propag. 61, 33 (2013).

[5] M. Silveirinha and N. Engheta, Phys. Rev. Lett. 97, 157403 (2006).

[6] A. Alu and N. Engheta, IEEE Trans. Antennas Propag. 58, 328 (2010).

[7] Y. Jin and S. He, Opt. Express 18, 16587 (2010).

[8] J. Yang, M. Huang, and J. Peng, Radioengineering 18, 124 (2009).

[9] V. C. Nguyen, L. Chen, and K. Halterman, Phys. Rev. Lett. 105, 233908 (2010).

[10] R. Fleury and A. Alù, Phys. Rev. B 87, 201101 (2013).

[11] Y. Li, I. Liberal, C. D. Giovampaola, and N. Engheta, Sci. Adv. 2, e1501790 (2016).

[12] I. Liberal and N. Engheta (to be published).

[13] M. A. Vincenti, D. de Ceglia, A. Ciattoni, and M. Scalora, Phys. Rev. A 84, 063826 (2011).

[14] H. Suchowski, K. O'Brien, Z. J. Wong, A. Salandrino, X. Yin, and X. Zhang, Science 342, 1223 (2013).

[15] T. S. Luk, D. de Ceglia, S. Liu, G. A. Keeler, R. P. Prasankumar, M. A. Vincenti, M. Scalora, M. B. Sinclair, and S. Campione, Appl. Phys. Lett. 106, 151103 (2015).

[16] A. Capretti, Y. Wang, N. Engheta, and L. D. Negro, Opt. Lett. 40, 1500 (2015)

[17] C. Argyropoulos, P.-Y. Chen, G. D'Aguanno, N. Engheta, and A. Alù, Phys. Rev. B 85, 045129 (2012).

[18] A. Ciattoni, C. Rizza, and E. Palange, Phys. Rev. A 81, 043839 (2010).

[19] N. Kinsey, C. DeVault, J. Kim, M. Ferrera, V. M. Shalaev, and A. Boltasseva, Optica 2, 616 (2015).

[20] L. Caspani, R. P. M. Kaipurath, M. Clerici, M. Ferrera, T. Roger, J. Kim, N. Kinsey, M. Pietrzyk, A. Di Falco, V. M. Shalaev, A. Boltasseva, and D. Faccio, Phys. Rev. Lett. 116, 233901 (2016).

[21] M. Z. Alam, I. De Leon, and R. W. Boyd, Science 352, 795 (2016).
[22] L. Parker, Phys. Rev. 183, 1057 (1969).

[23] N.D. Birrell and P.C.W. Davies, Quantum Fields in Curved Space (Cambridge University Press, Cambridge, 1984), Vol. 7.

[24] T. Jacobson, arXiv:gr-qc/0308048.

[25] L. H. Ford, arXiv:gr-qc/9707062.

[26] V. F. Mukhanov and S. Winitzki, Lecture notes in artificial intelligence 2004 (2007).

[27] V. F. Mukhanov and G. V. Chibisov, Pis'ma Zh. Eksp. Teor. Fiz. 33, 549 (1981) [JETP Lett. 33, 532 (1981)].

[28] J. Martin, in Planck Scale Effects in Astrophysics and Cosmology, J. Kowalski-Glikman and G. Amelino-Camelia (Springer Berlin Heidelberg, Berlin, Heidelberg, 2005), pp. 199-244.

[29] J. Martin, in Inflationary Cosmology, M. Lemoine, J. Martin, and P. Peter (Springer Berlin Heidelberg, Berlin, Heidelberg, 2007), pp. 193-241.

[30] C. Barceló, S. Liberati, and M. Visser, Int. J. Geom. Methods Mod. Phys. D12, 1641 (2003).

[31] C. Barceló, S. Liberati, and M. Visser, Phys. Rev. A 68, 053613 (2003).

[32] M. Uhlmann, Y. Xu, and R. Schützhold, New J. Phys. 7, 248 (2005).

[33] A. Prain, S. Fagnocchi, and S. Liberati, Phys. Rev. D 82, 105018 (2010).

[34] R. Schützhold, M. Uhlmann, L. Petersen, H. Schmitz, A. Friedenauer, and T. Schätz, Phys. Rev. Lett. 99, 201301 (2007).

[35] P. O. Fedichev and U. R. Fischer, Phys. Rev. A 69, 033602 (2004).

[36] S. Liberati, A. Prain, and M. Visser, Phys. Rev. D 85, 084014 (2012)

[37] J. T. Mendonça, A. Guerreiro, and A. M. Martins, Phys. Rev. A 62, 033805 (2000).

[38] J. T. Mendonça and A. Guerreiro, Phys. Rev. A 72, 063805 (2005).

[39] E. Yablonovitch, Phys. Rev. Lett. 62, 1742 (1989).

[40] R. W. Boyd, Nonlinear Optics (Academic Press, New York, 2003).

[41] S. Winitzki, Phys. Rev. D 72, 104011 (2005).

[42] See Supplemental Material http://link.aps.org/supplemental/ 10.1103/PhysRevLett.118.133904, which includes Ref. [43].

[43] P. Boonserm and M. Visser, J. High Energy Phys. 03 (2011) 073.

[44] A. A. Schulz, A. A. Tahir, M.Z. Alam, J. Upham, I. De Leon, and R. W. Boyd, Phys. Rev. A 93, 063846 (2016).

[45] J. Kim et al., Optica 3, 2334 (2016).

[46] D. Walls and G. Milburn, Quantum Optics (Springer, New York, 1994).

[47] U. Leonhardt, Essential Quantum Optics (Cambridge University Press, Cambridge, 2010). 